\title{
7. A Chromosome Study on the 91 Cases of the Mentally Retarded Men
}

\author{
By Tetsuji Kadotani, *) Yoko Watanabe,*) Hisaki Tanaka,**) \\ and Suzue KANATA***)
}

(Communicated by Sajiro Makıno, M. J. A., Jan. 13, 1986)

A chromosomal survey was done in the patients in Roppo-Gakuen, which was an establishment for the mentally retarded men, during a period from February 1981 to February 1983, 91 cases were collected for the chromosomal study.

The chromosome slides were prepared following the standard blood cultures. The karyotype analyses were made with the application of the conventional Giemsa staining, and G- and Q-banding differential staining.

Results and remarks. Table I showed the results of the clinical examinations and chromosomal findings. All patients had mental retardation; imbecile.

Eighteen cases out of ninety one cases were found to possess the abnormal karyotypes, being $19.8 \%$ in incidence. But the remaining seventy-three cases had the normal karyotype. And thirteen cases out of the abnormal eighteen cases were Down's syndrome, being $72.2 \%$ in incidence (Table I). The abnormalities were found at a little low level in incidence to those of the former survey; $23.5 \%$ in incidence in congenital defectives, ${ }^{1)} 26.2 \%$ in incidence in the patients such as a cerebral palsy and seizure.2)

They were represented by eighteen cases; one case of a reciprocal translocation between no. 7 and no. 14 chromosomes $(46, \mathrm{XY}, \operatorname{rcp}(7 ; 14)(\mathrm{q} 11 ; \mathrm{q} 22))$, one case of a deletion of no. 10 chromosome (46,XY,del(10) (p14)), one case of the translocation between no. 13 and no. 14 chromosomes $(45, \mathrm{XY},-13,-14,+\mathrm{t}(13 ; 14)$ (p11;p11)), one case of the ring chromosome of no. 18 (46,XX,r (18) (p11;q23)), eleven cases of Down's syndrome having trisomy no. 21 chromosomes (47, XX or $\mathrm{XY},+21)$, two cases of translocational Down's syndrome $(46, \mathrm{XX}, \mathrm{t}(14 ; 21))$, and one case having a very small marker chromosome $(47, \mathrm{XX},+$ mar $)$.

Acknowledgements. We are cordially obliged to Emeritus Professor Sajiro Makino, M. J. A., the senior director of the Kadotani Medical Research Foundation, for improvement of this manuscript. Financial aid from the Japan Academy is gratefully acknowledged here.

\section{References}

1) Kadotani, T. et al. (1982): Proc. Japan Acad., 58B, 89-92.

2) Kadotani, T. et al. (1984): ibid., 60B, 129-130.

\footnotetext{
*) The Kadotani Medical Research Foundation, 1248, Saijohigashi, Saijo, Higashihiroshima, Japan.

**) Roppo-Gakuen, Taguchi, Saijo-cho, Higashihiroshima, Japan.

***) Psychiatric Department, Kamo National Sanatorium, Kurose-cho, Kamogun, Hiroshima, Japan.
} 
Table I. Clinical and cytogenetic findings in 91 cases

\begin{tabular}{|c|c|c|c|c|}
\hline $\begin{array}{c}\text { Case } \\
\text { no. }\end{array}$ & Age & Sex & Karyotype & Clinical features \\
\hline 1. & $33 \mathrm{y}$. & $\mathrm{F}$ & $46, \mathrm{XX}$ & $\begin{array}{l}\text { dilatation of the heart, anemia, adrenal hyper- } \\
\text { tension, short stature, hypertelorism, low nasal } \\
\text { bridge, flat nasal base, small nose, flat breasts, } \\
\text { hypoplastic thumb }\end{array}$ \\
\hline 2. & $32 \mathrm{y}$. & $\mathrm{F}$ & $46, \mathrm{XX}$ & hirsutism \\
\hline 3. & $38 \mathrm{y}$. & $\mathbf{F}$ & $46, \mathrm{XX}$ & vagabondism \\
\hline 4. & $37 \mathrm{y}$. & $\mathrm{F}$ & $47, \mathrm{XX},+21$ & $\begin{array}{l}\text { Down's syndrome, short neck, flat facies, epican- } \\
\text { thus, upward slanted palpebral fissure, low } \\
\text { nasal bridge, saddle nose, flat nasal base, nar- } \\
\text { row high arched palate, hypoplastic thumb, V. } \\
\text { finger clinodactyly }\end{array}$ \\
\hline 5. & $23 y$ & $\mathrm{~F}$ & $46, \mathrm{XX}$ & $\begin{array}{l}\text { moon facies, flat nasal base, large nose, narrow } \\
\text { high arched palate }\end{array}$ \\
\hline 6. & $21 \mathrm{y}$. & $\mathrm{F}$ & $47, \mathrm{XX},+21$ & $\begin{array}{l}\text { Down's syndrome, fiat occiput, short neck, up- } \\
\text { ward slanted palpebral fissure, epicanthus, low } \\
\text { nasal bridge, saddle nose, flat nasal base, brachy- } \\
\text { dactyly }\end{array}$ \\
\hline 7. & $24 \mathrm{y}$. & $\mathrm{F}$ & $46, \mathrm{XX}$ & narrow high arched palate \\
\hline 8. & $24 y$. & $\mathrm{F}$ & $46, \mathrm{XX}$ & $\begin{array}{l}\text { short stature, narrow high arched palate, mis- } \\
\text { plasia and malinplantation in teeth, small } \\
\text { thorax of left, hypoplastic thumb, arachno- } \\
\text { dactyly }\end{array}$ \\
\hline 9. & $23 y$. & $\mathrm{F}$ & $47, \mathrm{XX},+\operatorname{mar}$ & $\begin{array}{l}\text { brain edema, epilepsy, short stature, narrow } \\
\text { high arched palate, nocturnal enuresis }\end{array}$ \\
\hline 10. & $26 \mathrm{y}$ & $\mathrm{F}$ & $46, \mathrm{XX}$ & slightly epilepsy, narrow high arched palate \\
\hline 11. & $23 y$. & M & $46, \mathrm{XY}$ & normal feature \\
\hline 12. & $34 \mathrm{y}$. & $\mathrm{F}$ & $46, \mathrm{XX}$ & flat occiput, exophthalmus, epicanthus \\
\hline 13. & $23 \mathrm{y}$ & $\mathrm{F}$ & $46, \mathrm{XX}$ & flat occiput, enlargement of external genitalia \\
\hline 14. & $33 y$. & M & $46, \mathrm{XY}$ & short stature, simianline of right hand \\
\hline 15. & $22 \mathrm{y}$. & M & $46, \mathrm{XY}$ & normal feature \\
\hline 16. & $34 \mathrm{y}$. & M & $46, \mathrm{XY}$ & normal feature \\
\hline 17. & $29 y$. & M & $46, \mathrm{XY}$ & normal feature \\
\hline 18. & $22 \mathrm{y}$ & M & $46, \mathrm{XY}$ & normal feature \\
\hline 19. & $38 \mathrm{y}$. & $\mathrm{F}$ & $46, \mathrm{XX}$ & $\begin{array}{l}\text { flat facies, double-eyelid, hypertelorism, ptosis } \\
\text { of eyelid }\end{array}$ \\
\hline 20. & $22 y$. & M & $47, \mathrm{XY},+21$ & $\begin{array}{l}\text { Down's syndrome, short stature, small head, flat } \\
\text { occiput, moon facies, upward slanted palpebral } \\
\text { fissure, epicanthus, low nasal bridge, flat nasal } \\
\text { base, high arched palate, brachydactyly, hypo- } \\
\text { tonia, simianline of both hands }\end{array}$ \\
\hline 21. & $24 \mathrm{y}$ & M & $46, \mathrm{XY}$ & $\begin{array}{l}\text { flat and moon facies, V. finger clinodactyly, } \\
\text { hypotonia, simianline of both hands }\end{array}$ \\
\hline 22. & $40 \mathrm{y}$ & M & $46, \mathrm{XY}$ & normal feature \\
\hline 23. & $22 \mathrm{y}$. & $\mathrm{F}$ & $47, \mathrm{XX},+21$ & $\begin{array}{l}\text { Down's syndrome, short stature, microcephaly, } \\
\text { short and web neck, flat and moon facies, older } \\
\text { facies, hypertelorism, upward slanted palpebral } \\
\text { fissure, ptosis of eyelid, epicanthus, low set ears, } \\
\text { malformed auricle, low nasal bridge, nose de- } \\
\text { pressed, flat nasal base, high arched palate, } \\
\text { V. finger clinodactyly, hypotonia, simianline of } \\
\text { both hands }\end{array}$ \\
\hline
\end{tabular}


Table I (continued)

\begin{tabular}{|c|c|c|c|c|}
\hline $\begin{array}{l}\text { Case } \\
\text { no. }\end{array}$ & Age & $\operatorname{Sex}$ & Karyotype & Clinical features \\
\hline 24. & $19 \mathrm{y}$. & $\mathrm{M}$ & $46, \mathrm{XY}$ & $\begin{array}{l}\text { short stature, dolichocephalia, high arched pal- } \\
\text { ate }\end{array}$ \\
\hline 25. & $35 \mathrm{y}$. & M & $46, \mathrm{XY}$ & normal feature \\
\hline 26. & $35 \mathrm{y}$. & M & $46, \mathrm{XY}$ & normal feature \\
\hline 27. & $34 \mathrm{y}$. & M & $46, \mathrm{XY}$ & stubby finger \\
\hline 28. & $22 \mathrm{y}$. & M & $46, \mathrm{XY}$ & $\begin{array}{l}\text { dolichocephalia, upward slanted palpebral fis- } \\
\text { sure, hirsutism, high arched palate }\end{array}$ \\
\hline 29. & $19 \mathrm{y}$. & $\mathrm{F}$ & $46, \mathrm{XX}$ & double-eyelid, flat nasal base, large nose \\
\hline 30. & $31 \mathrm{y}$. & $\mathbf{F}$ & $46, \mathrm{XX}$ & normal feature \\
\hline 31. & $24 \mathrm{y}$. & $\mathrm{F}$ & $46, \mathrm{XX}$ & $\begin{array}{l}\text { microcephaly, flat occiput, epicanthus, promi- } \\
\text { nent nasal bridge, hypoplastic thumb }\end{array}$ \\
\hline 32. & $19 \mathrm{y}$. & F & $46, \mathrm{XX}$ & $\begin{array}{l}\text { hirsutism, moon facies, hypertelorism, epican- } \\
\text { thus, large nose, hypoplastic thumb, V. finger } \\
\text { clinodactyly, hypertonicity }\end{array}$ \\
\hline 33. & $12 \mathrm{y}$. & M & $47, \mathrm{XY},+21$ & $\begin{array}{l}\text { Down's syndrome, flat occiput, short neck, flat } \\
\text { forehead, moon facies, hypertelorism, upward } \\
\text { slanted palpebral fissure, epicanthus, short and } \\
\text { coarse eyelashes, low set ears, malformed auri- } \\
\text { cle, protruding tongue, hypoplastic thumb, V. } \\
\text { finger clinodactyly }\end{array}$ \\
\hline 34. & $26 y$. & M & $46, \mathrm{XY}$ & $\begin{array}{l}\text { microcephaly, hypertelorism, upward slanted } \\
\text { palpebral fissure, prominent nasal bridge, in- } \\
\text { voluntary movement, ataxia }\end{array}$ \\
\hline 35. & $12 \mathrm{y}$. & M & $46, \mathrm{XY}$ & $\begin{array}{l}\text { macrocephaly, low set ears, prominent nasal } \\
\text { bridge, V. finger clinodactyly, hypotonia }\end{array}$ \\
\hline 36. & $20 \mathrm{y}$. & F & $46, \mathrm{XX}$ & $\begin{array}{l}\text { hirsutism, macrocephaly, moon facies, strabis- } \\
\text { mus of left, flat nasal base, hypoplastic thumb, } \\
\text { V. finger clinodactyly, ataxia }\end{array}$ \\
\hline 37. & $7 \mathrm{y}$. & M & $\begin{array}{l}46, X Y \\
\quad \text { rep }(7 ; 14) \\
\quad(q 11 ; q 22)\end{array}$ & $\begin{array}{l}\text { sparse hair, scanty hair of the scalp, dolicho- } \\
\text { cephalia, oxycephaly, flat occiput, hypertelorism, } \\
\text { low set and large ears, low nasal bridge, flat } \\
\text { nasal base, V. finger clinodactyly at both sides }\end{array}$ \\
\hline 38. & $11 \mathrm{y}$. & F & $46, \mathrm{XX}$ & tiny microcephaly, V. finger clinodactyly \\
\hline 39. & $12 \mathrm{y}$. & $\mathrm{F}$ & $46, \mathrm{XX}$ & $\begin{array}{l}\text { macrocephaly, dolichocephalia, hypertelorism, } \\
\text { epicanthus, low set and simple ears, flat nasal } \\
\text { bridge }\end{array}$ \\
\hline 40. & $17 \mathrm{y}$ & $\mathrm{F}$ & $46, \mathrm{XX}$ & $\begin{array}{l}\text { fiat occiput, mask-like facies, hypertelorism, } \\
\text { epicanthus, low set ears, flat nasal bridge, hypo- } \\
\text { tonia, simianline of left hand }\end{array}$ \\
\hline 41. & $18 \mathrm{y}$. & $\mathrm{F}$ & $47, \mathrm{XX},+21$ & $\begin{array}{l}\text { mask-like facies, hypertelorism, upward slanted } \\
\text { palpebral fissure, epicanthus, low set ears, low } \\
\text { nasal bridge, flat nasal base, small nose, hypo- } \\
\text { tonia, simianline of left, short limbs }\end{array}$ \\
\hline 42. & $15 \mathrm{y}$. & $\mathrm{F}$ & $\begin{array}{l}\text { 46, XX, r }(18) \\
\quad(\mathrm{p} 11 ; \mathrm{q} 23)\end{array}$ & flat nasal base, simianline of both hands \\
\hline 43. & $17 \mathrm{y}$. & $\mathrm{F}$ & $46, \mathrm{XX}$ & $\begin{array}{l}\text { flat occiput, moon facies, epicanthus, saddle } \\
\text { nose, flat nasal base, V. finger clinodactyly, } \\
\text { simianline of both hands, hypotonia }\end{array}$ \\
\hline 44. & $19 \mathrm{y}$ & $\mathrm{F}$ & $46, \mathrm{XX}$ & $\begin{array}{l}\text { flat occiput, hypotelorism, upward slanted pal- } \\
\text { pebral fissure, epicanthus, saddle nose, flat nasal } \\
\text { base, large alae nasi }\end{array}$ \\
\hline
\end{tabular}


Table I (continued)

\begin{tabular}{|c|c|c|c|c|}
\hline $\begin{array}{c}\text { Case } \\
\text { no. }\end{array}$ & Age & Sex & Karyotype & Clinical features \\
\hline 45. & $18 \mathrm{y}$ & $\mathrm{F}$ & $47, \mathrm{XX},+21$ & $\begin{array}{l}\text { Down's syndrome, microcephaly, flat occiput, } \\
\text { short neck, flat forehead, moon facies, upward } \\
\text { slanted palpebral fissure, ptosis of eyelid, epi- } \\
\text { canthus, low set ears, flat nasal base, narrow } \\
\text { high arched palate, hypoplastic thumb, V. finger } \\
\text { clinodactyly, simianline of left hand }\end{array}$ \\
\hline 46. & $21 y$. & $\mathrm{F}$ & $46, \mathrm{XX}$ & flat occiput, narrow high arched palate \\
\hline 47. & $20 \mathrm{y}$. & $\mathrm{F}$ & $47, \mathrm{XX},+21$ & $\begin{array}{l}\text { Down's syndrome, short stature, scanty hair of } \\
\text { the scalp, microcephaly, flat occiput, lines of } \\
\text { brow, upward slanted palpebral fissure, epi- } \\
\text { canthus, blepharophimosis, saddle nose, flat } \\
\text { nasal base, high arched palate, V. finger clino- } \\
\text { dactyly, simianline of right, hypotonia }\end{array}$ \\
\hline 48. & $16 \mathrm{y}$ & $\mathrm{F}$ & $46, \mathrm{XX}$ & $\begin{array}{l}\text { flat occiput, strabismus of both eyes, prominent } \\
\text { nasal bridge, high arched palate }\end{array}$ \\
\hline 49. & $20 \mathrm{y}$. & $\mathrm{F}$ & $\begin{array}{l}\text { 46, XX, } \\
\text { t }(14 ; 21)\end{array}$ & $\begin{array}{l}\text { Down's syndrome, scanty hair of the scalp, } \\
\text { microcephaly, flat occiput, upward slanted pal- } \\
\text { pebral fissure, epicanthus, low set ears, flat } \\
\text { nasal base }\end{array}$ \\
\hline 50. & $21 \mathrm{y}$ & $\mathrm{F}$ & $47, \mathrm{XX},+21$ & $\begin{array}{l}\text { Down's syndrome, microcephaly, flat occiput, } \\
\text { short neck, upward slanted palpebral fissure, } \\
\text { epicanthus, blepharophimosis, short and coarse } \\
\text { eyelashes, low set ears, nose depressed, flat } \\
\text { nasal base, small nose, high arched palate, } \\
\text { prominent labium inferius, V. finger clinodacty- } \\
\text { ly, simianlines of both hands, hypotonia }\end{array}$ \\
\hline 51. & $22 \mathrm{y}$. & $\mathrm{F}$ & $\begin{array}{l}46, \mathrm{XX} \\
\mathrm{t}(14 ; 21)\end{array}$ & $\begin{array}{l}\text { Down's syndrome, short stature, microcephaly, } \\
\text { flat occiput, upward slanted palpebral fissure, } \\
\text { epicanthus, short and coarse eyelashes, low set } \\
\text { ears, flat nasal base, high arched palate, promi- } \\
\text { nent labium inferius, brachydactyly, hypotonia }\end{array}$ \\
\hline 52. & $20 \mathrm{y}$. & $\mathrm{F}$ & $46, \mathrm{XX}$ & microcephaly, flat occiput, external strabismus \\
\hline 53. & $13 \mathrm{y}$. & $\mathrm{F}$ & $46, \mathrm{XX}$ & nose depressed \\
\hline 54. & $17 \mathrm{y}$ & $\mathrm{F}$ & $46, \mathrm{XX}$ & $\begin{array}{l}\text { macrocephaly, hardness of hearing, speech dis- } \\
\text { orders }\end{array}$ \\
\hline 55. & $23 y$. & $\mathrm{F}$ & $46, \mathrm{XX}$ & $\begin{array}{l}\text { short and coarse eyelashes, low nasal bridge, } \\
\text { flat nasal base, high arched palate }\end{array}$ \\
\hline 56. & $21 \mathrm{y}$ & $\mathrm{F}$ & $46, \mathrm{XX}$ & macrocephaly, long eyelashes \\
\hline 57. & $21 \mathrm{y}$. & $\mathrm{F}$ & $46, \mathrm{XX}$ & microcephaly \\
\hline 58. & $24 \mathrm{y}$ & $\mathrm{F}$ & $46, \mathrm{XX}$ & normal feature \\
\hline 59. & $18 \mathrm{y}$. & $\mathrm{F}$ & $46, \mathrm{XX}$ & normal feature \\
\hline 60. & $20 \mathrm{y}$. & $\mathrm{F}$ & $46, \mathrm{XX}$ & $\begin{array}{l}\text { hypertelorism, low nasal bridge, simianlines of } \\
\text { both hands }\end{array}$ \\
\hline 61. & $18 \mathrm{y}$ & $\mathrm{F}$ & $46, \mathrm{XX}$ & $\begin{array}{l}\text { dolichocephalia, low nasal bridge, prominent } \\
\text { labium inferius, small labium }\end{array}$ \\
\hline 62. & $14 \mathrm{y}$. & M & $46, \mathrm{XY}$ & malformed auricle \\
\hline 63. & $15 \mathrm{y}$ & M & $46, \mathrm{XY}$ & $\begin{array}{l}\text { hypertelorism, upward slanted palpebral fissure, } \\
\text { blepharophimosis, flat nasal base, simianline of } \\
\text { right }\end{array}$ \\
\hline 64. & $16 \mathrm{y}$ & M & $46, \mathrm{XY}$ & V. finger clinodactyly \\
\hline
\end{tabular}


Table I (continued)

\begin{tabular}{|c|c|c|c|c|}
\hline $\begin{array}{c}\text { Case } \\
\text { no. }\end{array}$ & Age & Sex & Karyotype & Clinical features \\
\hline 65. & $18 \mathrm{y}$ & M & $\begin{array}{l}45, \mathrm{XY} \\
\quad-13,-14 \\
\quad+\mathrm{t}(13 ; 14) \\
\quad(\mathrm{p} 11 ; \mathrm{p} 11)\end{array}$ & $\begin{array}{l}\text { macrocephaly, malformed auricle, high arched } \\
\text { palate }\end{array}$ \\
\hline 66. & $17 \mathrm{y}$ & M & $46, \mathrm{XY}$ & hypotelorism, malformed auricle \\
\hline 67. & $17 \mathrm{y}$. & $\mathrm{M}$ & $\begin{array}{l}\text { 46, XY, } \\
\text { del }(10)(\mathrm{p} 14)\end{array}$ & $\begin{array}{l}\text { macrocephaly, flat occiput, epicanthus, microtia, } \\
\text { low nasal bridge, flat nasal base }\end{array}$ \\
\hline 68. & $16 \mathrm{y}$ & $\mathrm{M}$ & $46, \mathrm{XY}$ & long hands, simianline of right \\
\hline 69. & $16 \mathrm{y}$ & M & $46, \mathrm{XY}$ & microcephaly, oxycephaly \\
\hline 70. & $16 \mathrm{y}$ & M & $46, \mathrm{XY}$ & $\begin{array}{l}\text { microcephaly, oxycephaly, flat occiput, upward } \\
\text { slanted palpebral fissure, epicanthus, large ear, } \\
\text { arachnodactyly }\end{array}$ \\
\hline 71. & $16 \mathrm{y}$ & M & $46, \mathrm{XY}$ & long stature, flat occiput, hypotelorism \\
\hline 72. & $17 \mathrm{y}$ & $\mathrm{M}$ & $46, \mathrm{XY}$ & $\begin{array}{l}\text { dolichocephalia, flat occiput, short neck, long } \\
\text { fingers }\end{array}$ \\
\hline 73. & $18 \mathrm{y}$. & M & $46, \mathrm{XY}$ & flat occiput, low set ears, large nose \\
\hline 74. & $18 \mathrm{y}$. & M & $46, \mathrm{XY}$ & $\begin{array}{l}\text { downward slanted palpebral fissure, malformed } \\
\text { auricle, long fingers, hypotonia }\end{array}$ \\
\hline 75. & $8 \mathrm{y}$. & M & $47, \mathrm{XY},+21$ & $\begin{array}{l}\text { Down's syndrome, scanty hair of the scalp, } \\
\text { microcephaly, flat occiput, short neck, flat facies, } \\
\text { hypertelorism, upward slanted palpebral fissure, } \\
\text { short and coarse eyelashes, low set ears, low } \\
\text { nasal bridge, saddle nose, flat nasal base, small } \\
\text { nose, brachydactyly, V. finger clinodactyly, } \\
\text { simianlines of both hands }\end{array}$ \\
\hline 76. & $9 \mathrm{y}$. & M & $46, \mathrm{XY}$ & $\begin{array}{l}\text { oxycephaly, head deformity, mouth opening, V. } \\
\text { finger clinodactyly }\end{array}$ \\
\hline 77. & $8 \mathrm{y}$. & M & $47, \mathrm{XY},+21$ & $\begin{array}{l}\text { Down's syndrome, scanty hair of the scalp, } \\
\text { microcephaly, flat occiput, short neck, moon } \\
\text { facies, hypertelorism, upward slanted palpebral } \\
\text { fissure, epicanthus, low set ears, malformed } \\
\text { auricle, low nasal bridge, nose depressed, saddle } \\
\text { nose, flat nasal base, small nose, mouth open- } \\
\text { ing, brachydactyly, simianlines of both hands, } \\
\text { hypotonia }\end{array}$ \\
\hline 78. & $14 \mathrm{y}$ & M & $46, \mathrm{XY}$ & $\begin{array}{l}\text { dolichocephalia, downward slanted palpebral } \\
\text { fissure, mouth opening, long fingers, simianline } \\
\text { of left }\end{array}$ \\
\hline 79. & $16 \mathrm{y}$. & M & $46, \mathrm{XY}$ & $\begin{array}{l}\text { macrocephaly, oxycephaly, short neck, flat } \\
\text { facies, hypotelorism, epicanthus, short and } \\
\text { coarse eyelashes, blepharitis, low set ears, mal- } \\
\text { formed auricle, low nasal bridge, nose de- } \\
\text { pressed, saddle nose, flat nasal base, large nose, } \\
\text { mouth opening, protruding tongue, polydactyly }\end{array}$ \\
\hline 80. & $10 \mathrm{y}$ & M & $46, \mathrm{XY}$ & $\begin{array}{l}\text { macrocephaly, oxycephaly, flat occiput, down- } \\
\text { ward slanted palpebral fissure, low nasal bridge, } \\
\text { saddle nose, large nose }\end{array}$ \\
\hline 81. & $16 \mathrm{y}$. & M & $46, \mathrm{XY}$ & $\begin{array}{l}\text { macrocephaly, low set ears, malformed auricle, } \\
\text { misplasia and malinplantation in teeth, epilepsy }\end{array}$ \\
\hline 82. & $8 \mathrm{y}$. & $\mathrm{M}$ & $46, \mathrm{XY}$ & hypertelorism, high arched palate \\
\hline 83. & $15 \mathrm{y}$. & $\mathrm{M}$ & $46, \mathrm{XY}$ & macrocephaly, moon facies, high arched palate \\
\hline
\end{tabular}


Table I (continued)

\begin{tabular}{|c|c|c|c|c|}
\hline $\begin{array}{l}\text { Case } \\
\text { no. }\end{array}$ & Age & Sex & Karyotype & Clinical features \\
\hline 84. & $14 \mathrm{y}$. & M & $46, \mathrm{XY}$ & $\begin{array}{l}\text { dolichocephalia, hypotelorism, large nose, high } \\
\text { arched palate }\end{array}$ \\
\hline 85. & $20 \mathrm{y}$. & M & $46, \mathrm{XY}$ & $\begin{array}{l}\text { hypotelorism, malformed auricle, large nose, } \\
\text { high arched palate, simianline of right }\end{array}$ \\
\hline 86. & $13 \mathrm{y}$. & M & $46, \mathrm{XY}$ & $\begin{array}{l}\text { dolichocephalia, epicanthus, prominent nasal } \\
\text { bridge }\end{array}$ \\
\hline 87. & $18 \mathrm{y}$. & $\mathrm{M}$ & $46, \mathrm{XY}$ & $\begin{array}{l}\text { microcephaly, head deformity, hypotelorism, } \\
\text { epicanthus, malformed auricle, prominent nasal } \\
\text { bridge, beaked nose, high arched palate }\end{array}$ \\
\hline 88. & $8 \mathrm{y}$. & M & $46, \mathrm{XY}$ & $\begin{array}{l}\text { hypertelorism, epicanthus, low nasal bridge, } \\
\text { nose depressed }\end{array}$ \\
\hline 89. & $22 \mathrm{y}$ & M & $46, \mathrm{XY}$ & $\begin{array}{l}\text { microcephaly, epicanthus, low nasal bridge, nose } \\
\text { depressed, large nose, idiot }\end{array}$ \\
\hline 90. & $13 \mathrm{y}$. & M & $46, \mathrm{XY}$ & $\begin{array}{l}\text { scanty hair of the scalp, dolichocephalia, flat } \\
\text { occiput, hypertelorism, epicanthus, low nasal } \\
\text { bridge, large nose }\end{array}$ \\
\hline 91. & $18 \mathrm{y}$. & M & $46, \mathrm{XY}$ & high arched palate \\
\hline
\end{tabular}

